\title{
Clinical Features and Epidemiological Patterns of Infections by Multidrug Resistance Staphylococcus Aureus and Pseudomonas Aeruginosa in Patients with Burns
}

\author{
Abid Rashid ${ }^{1}$, Muhammad Akram² ${ }^{2}$ Omowumi T Kayode ${ }^{3}$ and Abolanle AA Kayode ${ }^{4 *}$ \\ ${ }^{1}$ Directorate of Medical Sciences, Government College University Faisalabad, Pakistan \\ ${ }^{2}$ Department of Eastern Medicine, Government College University Faisalabad, Pakistan \\ ${ }^{3}$ Department of Biochemistry, Landmark University, P.M.B 1001, Omu Aran, Kwara State, Nigeria \\ ${ }^{4}$ Department of Biochemistry, Benjamin S Carson (Snr) School of Medicine, Babcock University, Ilishan Remo, Ogun State, Nigeria \\ *Corresponding author: Abolanle AA Kayode, Department of Biochemistry, Benjamin S Carson (Snr) School of Medicine, Babcock \\ University, Ilishan Remo, Ogun State, Nigeria
}

\section{ARTICLE INFO}

Received: 幽 January 30, 2020

Published: 幽 February 12, 2020

Citation: Abid Rashid, Muhammad Akram, Omowumi T Kayode, Abolanle AA Kayode. Clinical Features and Epidemiological Patterns of Infections by Multidrug Resistance Staphylococcus Aureus and Pseudomonas Aeruginosa in Patients with Burns. Biomed J Sci \& Tech Res 25(4)-2020. BJSTR. MS.ID.004222.

Keywords: Clinical Features; Staphylococcus Aureus; Pseudomonas Aeruginosa; Patients with Burns

\section{ABSTRACT}

Background: Burn injuries are the most overpowering of all wounds and all around the world a major public health crisis. Burn wounds are the fourth most basic kind of injury around the world, after automobile accidents, falls, and interpersonal violence. About $90 \%$ of the burns happen in low to middle-class population of low socioeconomic countries.

Objectives: This paper aims to review the bacteria causing burn infections and their antimicrobial resistant profiles in individuals suffering from infected burn wounds.

Methods: Literature was obtained from different database such as Google Scholar and PubMed etc.

Results: Patients who endure serious consume are at the higher hazard for nearby and foundational diseases. As of late, rising safe pathogens have constrained consume mind suppliers worldwide to scan for elective types of treatment. The protection among different microbial species (irresistible operators) to various antimicrobial medications has developed a reason for general wellbeing risk around the world at a frightening rate. All the competent tainting specialists (e.g., microscopic organisms, growths, infection, and parasite) have utilized large amounts of Multi Drug Protection (MDR) with improved dismalness and mortality; along these lines, they are alluded to as "super bugs".

Conclusion: Although, the advancement of MDR is a characteristic marvel, the improper utilization of antimicrobial medications, insufficient clean conditions, unseemly sustenance dealing with, and poor contamination counteractive action and control hones add to development of and empower the further spread of MDR.

\section{Introduction}

Burns are more prevalent devastating type of trauma and require a critical care as well as handling. Mortality due to burns can be reduced by fluid replacement, excision, and coverage of burn wounds, infection treatment, and early resuscitation ShiraniVaughan et al. [1-6]. A better result for rigorously burned individuals has been credited to medical evolution in burn wound care, pulmonary care, nutritional support, fluid resuscitation and infection management. As a consequence, burn mortality, depending on the degree of a wound, has been decreased (up to $50 \%$ ) within the last decades Saffle Davis et al. [7,8]. $75 \%$ of all death in individuals with 
severe burns (over more than $40 \%$ of the total body surface area (TBSA) is affected), are linked to sepsis developed from infection of burn wounds or inhalation injury and / or other complications Fitzwater Purdue et al. [9-13]. Based on the preceding lines, it is very necessary to identify and isolate the multiple drugs=resistant bacteria, which will be very helpful to identify and manage the burn-wound infections in hospitals. The burns are totally different from other types of burn wounds on the body, such as the degree of systemic inflammation [14], healing of all burn wounds is an active procedure with overlapping stage Gurtner Werner et al. [15]. The early inflammatory stage brings monocytes and neutrophils to the place of damage via fluid extravasation and localized vasodilation, thus starting an immune response that is afterward continued by the macrophage's recruitment by chemokines Werner, Krieg et al. $[16,14]$. Despite aggressive burn wound care and a huge number of available antibiotics in the armamentarium of antimicrobials, burn wounds infections are predominant reason for death in patients with a burn. Of the variety of pathogens, multidrug resistant $\mathrm{S}$. aureus and Pseudomonas aeruginosa are most serious nosocomial pathogens in burn facilities worldwide.

\section{Multidrug Resistant}

Multidrug resistant is among the main three threats to global public health and is usually caused by substandard pharmaceuticals, excessive drug usage or prescription and inappropriate use of antimicrobials Santajit [17].

\section{Staphylococcus Aureus (MRSA)}

The sensitivity of isolated strains was against vancomycin, clindamycin, Kanamycin and Erythromycin, but high resistant was found against penicillin G. Multi drug resistance was found in all isolated strains while one isolated strain was found resistant to all the antibiotics Alebachew Yismaw et al. [18]. About 7 isolates of Staphylococcus epidermidis (S. epidermidis), 60 isolates of S. aureus were isolated from 540 wound and burns swabs from patient of cancer in Egypt. In 355 burn and surgical wound infected patients, 23 isolates of Klebsiella pneumonia, 4 isolates of Streptococcus pyogenes, 25 isolates of Escherichia coli, 27 isolates of Proteus vulgaris were identified. High resistance was found in all bacterial isolates against frequently used b-lactams (vancomycin, cefaclor amoxicillin, ampicillin, amoxicillin / clavulanic acid). The isolated bacterial strains showed little resistance to ciprofloxacin and imipenem. On plasmid analysis 2 susceptible and 6 multidrug resistant bacterial isolates showed same pattern [19]. Twentytwo strains of Pseudomonas aeruginosa, 8 strains of Klebsiella pneumonia, 17 strains of S. aureus, 6 strains of S. epidermidis, 7 strains of Acinetobacter baumannii and 5 strains of Enterobacter cloacae were isolated from burn wounds in a burn unit in a study conducted in Iraq.

Extended spectrum beta-lactamase production was seen in high proportion of Enterobacteriaceae. While methicillin-resistant against S. aureus isolates were also detected Ronat Kakol et al. [20]. Burn wound infections were polymicrobial in $26.6 \%$ of cases. Staphylococcus aureus was the most common aerobic isolate (32.4\%). Among S. aureus, 76.5\% were MDR strains, 73.5\% were methicillin-resistant, and $17.6 \%$ were vancomycin resistant. Coagulase-negative staphylococci (CoNS) were represented by $16.2 \%$. About $47 \%$ of CoNS strains were MDR strains, $47 \%$ were methicillin-resistant CoNS, and $23.6 \%$ were vancomycin-resistant CoNS. Multidrug resistance was seen in all Gram-negative bacteria isolated from burn wounds. Extended-spectrum b-lactamases production was observed in 58.5\% isolated strains, whereas metallo-b-lactamases production was detected in $43.4 \%$ of Gramnegative bacteria. Young age, low socioeconomic status, abuse of invasive procedures and antimicrobial, prolonged hospital stay, and improper control of burn-associated comorbidities were the risk factors for burn wound infections Melake et al. [21].

Thirty burn wound swab isolates obtained from 14 pediatric burns patients were investigated. Cultures were done on the first day for all patients and empirical antibiotic administration was started for those with septic burns with piperacillin-tazobactum and vancomycin. Microbial identification and antimicrobial sensitivity testing were done for all 30 isolates. The predominant bacteria were Pseudomonas aeruginosa, Acinetobacter, and Staphylococcus aureus. Most of the Acinetobacter and Staphylococcus aureus showed multidrug resistance. The Tissue Culture Plate (TCP) method was used to study the production of biofilm for all bacterial isolates, and results showed that most of the MDR isolates formed biofilm Ramakrishnan et al. [22].

\section{Methicillin Resistant Staphylococcus Aureus (MRSA) Mechanisms of Antibiotic Resistance in MRSA}

Primarily, several strains of MRSA were considered to possess a single common ancestor due to the various pigmentations than those of MSSA strains. However, the precise mechanism of transfer of gene (mecA gene) which is responsible for antibiotic resistance remains controversial [23]. In the evolution of S. aureus, horizontal transfer has main part, however the horizontal transmission of staphylococcus cassette chromosome element is rare Kuroda et al. [24]. MRSA is commonly found in hospital environment and chances of acquiring this pathogen are increased dramatically after the course of antibiotics. Antimicrobial medicines that are not effective against MRSA increase the likelihood of acquiring MRSA. However, some antibiotic groups like cephalosporin and quinolones are recognized as a potential suspect. Crowcroft et al. $[25,26]$. In humans, MRSA has been identified amongst the most intimidating and significant bacteria that because hospital acquired infections. A number of mechanisms has been recognized that how MRSA develop resistance against methicillin. The chief factor in antibiotic resistance is its capability to synthesize specific binding protein (PBP-2a) that renders $\beta$ lactam drugs ineffective and all $\beta$-lactamase resistant penicillin Chambers [27]. 


\section{MRSA Infections in Humans}

In human, S. aureus causes a variety of diseases. It is amongst one of the major etiologies of skin and soft tissues infections like bullous impetigo, cellulitis, furuncles, abscess, carbuncles, and folliculitis. Majority of these infections are resolved within few weeks and does not require any treatment. However, some of the antibiotic treatment is along with incision and drainage. And if they are left untreated, serious consequences may results, i.e. septic shock Lina et al. [28] or septicemia. S. aureus is also linked with deep-rooted infections like endocarditis and osteomyelitis Lindenmayer et al. [29]. Animals and humans are the normal reservoir of S. aureus. And it colonizes themselves, primarily in their nares. Apart from this location, S. aureus also present in groin, perineum, throat, and skin. It has been stated that a wide range (10 to $40 \%$ ) of the affected population with S. aureus does not have any hospital exposure. Anterior nares and throat were the predominant sites for this organism in these cases [30]. Various studies prove that in critically ill patient's MRSA nasal carriers has the major role in the development of disease. In general population as well in hospital, the major cause of infections is due to nasal colonization of this microorganism Wertheim et al. [31,32]

Different conditions and diseases in human like skin diseases, diabetes mellitus, AIDS and Intravenous drug abuse are related to higher nasal carriage rates of S. aureus and results in increased frequency of diseases Luzar et al. [33,34]. Other than soft tissue and skin infections, S. aureus infect joint and bone. It may result in pneumonia, scalded skin syndrome, and endocarditis. Hospitalization is necessary in severe cases of S. aureus and sometimes it requires antibiotic therapy through IV. In Europe, USA and Canada S. aureus is the most commonly isolated microorganisms, while throughout the world pneumonia and blood stream infections caused by S. aureus are more prevalent Diekema et al. [35]. In Finland a study reported that annually 700-900 cases of septic infection were reported that are caused by S. aureus Lyytikäinen et al. [36]. Cather related and post-operative infections are frequently caused by S. aureus. Catheter associated diseases are designated as the most common source of nosocomial bacteremia [37]. Toxic Shock Syndrome (TSS) is an acute disease that effect many organ systems and is caused by S. aureus. The clinical manifestations of disease are desquamation of skin, hypotension, multiple organ dysfunction and high-grade fever Dings et al. [38].

\section{Epidemiology of Human MRSA Infections}

Most of the infections occurring in health care facilities and in community are due to MRSA Drews et al. [39] Throughout the world it was reported that $\mathrm{S}$. aureus has shown resistance to various drugs particularly to fluroquinolones, aminoglycosides, macrolides, methicillin, lincosamides, or mixtures of all these Deshpande et al. [40] Earlier researches from United State of America, Japan, United Kingdom and Australia have stated that prevalence of MRSA vary from 8 - $53 \%$ [41]. MRSA has a worldwide distribution and has a great impact on mortality rate. In a meta-analysis of thirty studies, average mortality rate of methicillin susceptible S. aureus (MSSA) was $24 \%$ as compared to $36 \%$ of MRSA. Seven studies in this metaanalysis represent a mortality rate of $50 \%$ whereas two researches showed mortality rate of $80 \%$ Kuikka et al. [42,43] Vancomycin resistant strains (VRSA) also has a lethal effect Fridkin et al. [44] Patient infected with vancomycin intermediate S. aureus (VISA) has a mortality rate of $63 \%$ Fridkin et al. [45].

In hospitals of USA, MRSA was appeared to be the most prevalent pathogen that is responsible for antibiotic resistance (NNIS, 2004). During 1992-2003 in intensive care units (ICU) of USA the prevalence of MRSA were raised up to $28.5 \%$ Klevens et al. [46] In United Kingdom (UK) similar upsurge in prevalence of MRSA was noted, that raised from 2 to $43 \%$ from 1990 to 2002 Johnson et al. [47] In another study, mortality rate was doubled in MRSA bacteremia as compared to MSSA. In the same way, MRSA infected patients has a prolong duration of stay in hospital which results in increased expenditure of the treatment. This is due to extra cost on prolonged hospitalization, laboratory test and antibiotics that increase the economic burden Björholt [48]. In hospitals of Pakistan a frightening increase in MRSA has been noted Hafiz et al. [49] In Pakistan from 1996 to 2003, among isolates of nosocomial infections rates of MRSA raised from $39 \%$ to $51 \%$ Butt et al. [50] Another study conducted in a local hospital of Pakistan showed $38.5 \%$ cases of MRSA. In a hospital of Karachi, a study was conducted, which showed $22 \%$ cases of MRSA among 850 isolate of S. aureus Hakim et al. [51] In Gujranwala a study showed prevalence of hospital acquired MRSA (HA-MRSA) was $72.91 \%$, whereas community acquired MRSA (CA-MRSA) was $64.35 \%$. In skin and soft tissues infections the prevalence of HA-MRSA was $68.57 \%$ and that of CA-MRSA was $26.6 \%$ Ahmed et al. [52]

\section{MRSA Treatment in Humans}

Septicemia caused by vancomycin / rifampicin resistance strains of staphylococcus has a mortality of higher than $78 \%$ Burnie et al. [53] This high rate of mortality and advancement in the resistance patterns shows the conditions of last sixty years and future concerns. It has been recommended that all over the world, widespread prevalence of MRSA strains are the main reasons for increased mortality rate. To fight these pathogens, new medicines are introduced continuously. But no assurance can be given that these drugs are $100 \%$ effective as required. Despite of proper treatment, a number of reports showed that MRSA is associated with poor consequences Blot et al. [54] Empirical antibiotic therapy is usually given to the patients of bacteremia without any identification and sensitivity test. Such treatment strategies further increases the resistance capability of organism and make it difficult to manage the infection Harbarth et al. [55] In the case of MRSA infections, this has been well recognized as only twenty-five percent of the patients receive appropriate antimicrobial treatment with in forty-eight hours of hospital admission. And after 48 hours, only 
$40 \%$ of the patients received precise medicines. Various studies prove that in clinical practices wrong antimicrobial medication plays an important role in the generation of resistance to these drugs. Whereas, appropriate antibiotics if given in low doses, have similar outcome. A number of virulence factors are expressed by MRSA, which are responsible for treatment failure and even results in death of the patient occurs if appropriate antibiotic treatment is not started in 1st attempt. Antibiotic having beta-lactam group and their derivatives, i.e. cephalosporins and penicillins have no effect on MRSA. This is mainly due to the production of $\beta$-lactamase enzymes and normal Penicillin Bindings Proteins (PBPs). In clinical set ups Borderline Resistant S. aureus (BORSA) occasionally because threat, but distinguishing MRSA from BORSA strains still remains a challenge. However, majority of $\mathrm{S}$. auerus infections are treated by penicillin G. However, a strain of S. aureus is also present which produces $\beta$-lactamase, a penicillinase. In various part of the world, this resistance pattern was present.

In 1960 this resistance lead to the discovery of new medicine that are resistant to penicillinase i.e. cloxacillin, nafcillin, oxacillin and methicillin. In hospitals during subsequent decades, MRSA has emerged as serious hazards for health care system. Many antibiotics like tetracycline, lincosamides, fluroquinolones, are macrolides aminoglycosides were ineffective against HA-MRSA For the treatment of HA-MRSA vancomycin are used as alternative. However resistant against this microorganism was also reported Kuehnert et al. [56] CA-MRSA as compare to HA-MRSA has a fixed pattern of sensitivity to various $\beta$-lactam antibiotics like tetracycline Zetola et al. [57] clindamycin and TMP-SUX [58]. This variation pattern of resistance in nosocomial isolates of CA-MRSA is mainly due to the absences of SSCmec type IV. For the treatment of CA-MRSA infections, TMP-SUX is widely used. A study found that around $95 \%$ of CA-MRSA isolates were sensitive to TMP-SMX. However, some resistance was also observed [59].

\section{Pseudomonas Aeruginosa}

Pseudomonas aeruginosa is a leading cause of healthcare associated infection especially in admitted patients with burn [60]. It is an opportunistic pathogen and can survive in the hospital environment. It is associated with increased morbidity and mortality in immunosuppressed patients [61]. According to a study, the most prevalent organism isolated from patients with burn was P. aeruginosa (54.9\%). Similarly, other studies showed a prevalence of $P$. aeruginosa infection among patients with burn to be $53.97 \%$ and $59.6 \%$ and $37.5 \%$ respectively Arslan et al. [62-64] A breach in the protective skin barrier, reduced immunity, and prolonged hospital stay are important factors responsible for infection of burn wound with such opportunistic pathogens especially multi-drug resistant (MDR) P. aeruginosa Naqvi et al. [63] The presence of dead, denatured tissues and moist environment makes the burn wound vulnerable to infection by $P$. aeruginosa. Patients and their relatives are important sources of $P$. aeruginosa in ICU or other critical care units and have become a potential source of healthcare-associated infections. P. aeruginosa is increasingly becoming resistant to many anti-pseudomonal agents and is naturally resistant to many antibiotics. Limited treatment options are available for patients with burn infected with $P$. aeruginosa Ikpeme et al. [64]. Pseudomonas aeruginosa is naturally resistant to penicillin and most of the $\beta$-lactam antibiotics [65] P. aeruginosa utilize majority detecting to prompt the generation of destructiveness factors, for example, proteases, hemolysins, exotoxin An and pyocyanin. These microscopic organisms have two majority detecting frameworks: one that manages proteases and another that directs hemolysins. In spite of the fact that different frameworks, the two connect through results of the protease framework controlling the Hemolysins framework at both transcriptional and posttranslational levels. While it has been generally held that majority detecting is required for biofilm development, new research has been advanced that difficulties that position. Groups of researchers out of Auburn University explored the improvement of $P$. aeruginosa biofilms utilizing a mouse consume demonstrate. Third-degree warm damage was instigated, and mice were then contaminated with two strains of $P$. aeruginosa, one Wild Compose (WT) and the other majority detecting inadequate (QS). Eschar tests were inspected utilizing fluorescence and examining electron microscopy at 8, 24, and $48 \mathrm{~h}$ after contamination.

The two strains created biofilms at a similar pace, in spite of the fact that WT biofilms were to some degree denser than the QS strain. It had been forehanded exhibited that the WT strain was more destructive than the QS. This investigation proposes that the distinction in destructiveness is not because of weakened biofilms arrangement; rather it is likely because of contrasts in the statement of harmfulness factors. It appears that majority detecting knockout strains lost some different qualities during the time-spent change. Proteases are destructiveness factors that corrupt the trustworthiness of the host's physical boundaries by part proteins and amino acids, taking into account further penetration of disease. Exotoxin An ends the amalgamation of proteins, causing neighborhood tissue harm, immunosuppression and cell passing. Hemolysins act like cleansers to separate lipids in epithelial cells permitting, similar to the proteases, the bacilli to enter the host bringing about the spread of disease. Furthermore, qualities encoding proteins for pili and flagella are likewise vital to $P$. aeruginosa since without these structures, microscopic organisms cannot explore their condition and shape biofilms. $P$. aeruginosa diseases are hard to treat in light of the fact that these microorganisms have various systems for evading predators, including multidrug efflux pumps, anti-infection changing catalysts, and intense external layers with low penetrability.

P. aeruginosa infections are problematic due to its intrinsic as well as acquired resistance to many effective groups of antibiotics. Intrinsic MDR Pseudomonas aeruginosa is attributed by limited permeability of outer membrane, production of inducible $\beta$ - 
lactamase and Multidrug Efflux system. Among four MDR efflux systems in P. aeruginosa, MexXY-OprM and MexAB-OprM contribute to intrinsic resistance while hyper-expression of MexEF-OprN and MexCD-OprJ leads to acquired MDRPa. Plasmid and integrin have a crucial role in acquisition of mobile elements Upadhaya et al. [66] Around one hundred and thirty three P. aeruginosa disconnects were gathered from copy patients. $88.7 \%$ separated strains were from wounds took after by $5.26 \%$ detaches from blood, $4.15 \%$ from subclavian catheters and $1.5 \%$ from pee test. The anti-infection vulnerability profiles were finished by the agar circle dispersion. The outcomes demonstrated that protection against carbenicillin was 99.2\%, 98.4\% against ticarcillin, 96.2\% against ciprofloxacin, $95.4 \%$ against co-trimoxazole, $94.7 \%$ against imipenem and meropenem, 93.9\% against piperacillin, 93.2\% against aztreonam, $92.4 \%$ against tobramycin, $91.7 \%$ against cefepime, 89.4\% against amikacin and ceftazidime, lastly $87.2 \%$ against piperacillin-tazobactum. Generally, all the secluded strains indicated multidrug (protection from $\geq 3$ classes of anti-toxins) including the imipenem-safe detaches [59]. In patients with burn, fifty-six strains of $P$. aeruginosa were isolated. Aminoglycosides resistance was found in 58 (81\%) P. aeruginosa strains. While $41-70 \%$ isolated strains had resistance against beta-lactamspiperacillin, ceftazidime, and aztreonam. Piperacillin-tazobactum resistance was found in $34.5 \%$ while $12.06 \%$ strains were found to be resistant against ciprofloxacin. About $13-19 \%$, isolates were resistant to carbapenems. Celestin was found sensitive against all strains. P. aeruginosa was found to be resistant to three of the four 'in-use' drugs i.e. piperacillin+ tazobactam, ceftazidime, imipenem and gentamicin, which was taken as MDR, which depicted MDR percentage as $36.2 \%$ Biswal et al. [67] Seventeen P. aeruginosa were isolated from 100 burn wound infected patients in India. Multidrug resistance was found in all strain of $P$. aeruginosa. The resistance profile of the tested antibiotics was as done by using Kirby Bauer's Disc Diffusion Method. All the isolates were resistant to Tobramycin. While resistance against Meropenem was $94.1 \%$, against Cefoperazone was $94.1 \%$.

The resistance against other tested antibiotics was as Imipenem (88.2\%), Piperacillin/Tazobactum (82.4\%), Cefotaxime (76.5\%), Ceftazidime (70.6\%), Norfloxacin (70.6\%),Cefepime (64.7\%), Amikacin (47.1\%),Gentamicin (47.1\%) and Ciprofloxacin (35.5\%) Upadhaya et al.[66]. The most common bacterial microbes of burn wounds in Pakistan Institute of Medical Sciences (PIMS) were $P$. aeruginosa, K. pneumoniae, and S. aureus. The infections with positive cultures were found more frequently among those patients who had about more than two weeks duration of burn wounds. They found that the burden of burn wound infection can be reduced by skin grafting of deep burns wound, early excision and adherence to infection control measures Saaiq et al. [68] Fifty isolates of $P$. aeruginosa were identified among 250 burn wound children samples. The isolates of Pseudomonas aeruginosa had high resistance against ceftazidime (86\%), and cefotaxime (72\%) while no resistance was found to imipenem. Twenty-eight isolates (56\%) were multidrug resistant and $71.4 \%$ of them had integrin class 1 . ESBL was detected in $51 \%$ of the ceftazidime resistant strains of which only $12.5 \%$ harbored blaTEM gene and none had blaSHV gene Hassuna et al. [69]

Twenty-three examinations $(7,881$ patients with vulnerable $P$. aeruginosa, 1,653 with safe $P$. aeruginosa, 559 with MDR $P$. aeruginosa, 387 non-contaminated patients without $P$. aeruginosa) were examined. Arbitrary impacts demonstrate meta-examination was achievable for the endpoint of all-cause in-clinic mortality. Allcause mortality was 34\% (95\% certainty interim (CI) 27\% - 41\%) in patients with any safe $P$. aeruginosa contrasted with $22 \%$ (95\% CI $14 \%-29 \%$ ) with vulnerable $P$. aeruginosa. The meta-investigation showed a $>2$-crease expanded danger of mortality with MDR $P$. aeruginosa (relative hazard (RR) 2.34, 95\% CI 1.53 - 3.57) and a 24\% expanded hazard with safe P. aeruginosa (RR 1.24, 95\% CI 1.11 -1.38), contrasted with defenseless P. aeruginosa. A balanced metaexamination of information from seven investigations showed a factually non-huge expanded danger of mortality in patients with any safe P. aeruginosa (balanced RR 1.24, 95\% CI 0.98 - 1.57). Every one of the three examinations that revealed contamination related mortality found a factually fundamentally expanded hazard in patients with MDR $P$. aeruginosa contrasted with those with vulnerable $P$. aeruginosa. Crosswise over investigations, healing center length of stay (LOS) was higher in patients with safe and MDR $P$. aeruginosa contaminations, contrasted with helpless $P$. aeruginosa and control patients. Constraints included heterogeneity in MDR definition, confinement to nosocomial contaminations, and potential bewildering in examinations. Antimicrobial susceptibility testing was carried out for 56 isolates of P. aeruginosa by KirbyBauer disk diffusion method. The antibiogram of these isolates, 45 $(80.36 \%)$ isolates were resistant to piperacillin and 37 (66\%) were resistant to the piperacillin-tazobactum combination. 43 isolates (77\%) showed resistance to the anti-pseudomonal cephalosporin, ceftazidime and $36(65 \%)$ isolates were found to be resistant to cefepime. Among the aminoglycosides, 41 (73\%) isolates were found to be resistant to amikacin, 47 (84\%) to gentamicin and 42 $(75 \%)$ isolates were resistant to tobramycin.

A total $40(71 \%)$ isolates were resistant to ciprofloxacin and $26(47 \%)$ were resistant to levofloxacin. Imipenem resistance was seen in $34(61 \%)$ isolates whereas 30 (54\%) isolates were found to be resistant to meropenem. The retrospective study evaluated bacteria from sputum, urine, feces, blood, catheters, and wounds of hospitalized patients of burn. Resistance of $P$. aeruginosa and quantity of antibacterial medicines to anti gram-negative antibiotics were observed. Annual detection rate of S. aureus were declined significantly, as compare to Klebsiella pneumonia and $P$. aeruginosa whose rates were significantly raised. MDR strains of $P$. aeruginosa were also increasing day by day. Rate of resistance of P. aeruginosa is positively correlated to the intensity of use of the antimicrobials. Extra care should be given to K. pneumoniae and $P$. 
aeruginosa in burn wards. To counter emergence of resistance, use of cefoperzone/salbactam ceftazidime, and ciprofloxacin should be avoided Song et al. [70].

\section{Conclusion}

In conclusion, Innovations and advancements in liquid administration, ventilatory help, surgical care, and antimicrobial treatment have added to real advance in burn care and a huge decrease in related mortality and morbidity rates. Methicillinresistant $\mathrm{S}$. aureus-related and $P$. aeruginosa diseases remain a test because of the rise of more safe strains and the changing range of accessible medications. The advancement of new specialists, reasonable and proper utilization of anti-biotics agents, and better contamination control conventions are vital in the proceeding with fight against multi-resistant organisms.

\section{References}

1. Shirani KZ, GM Vaughan, AD Mason, BA Pruitt (1996) Update on current therapeutic approaches in burns. Shock (Augusta Ga.) 5(1): 4-16.

2. Altoparlak U, S Erol, MN Akcay, F Celebi, A Kadanali, et al. (2004) The time-related changes of antimicrobial resistance patterns and predominant bacterial profiles of burn wounds and body flora of burned patients. Burns 30(7): 660-664.

3. Pham TN, LC Cancio, NS Gibran (2008) American Burn Association practice guidelines burn shock resuscitation. J Burn Care Res 29(1): 257-266.

4. Dries DJ (2009) Management of burn injuries-recent developments in resuscitation, infection control and outcomes research. Scandinavian j trauma, resuscitation emergency med 17(1): 14

5. Nisanci M, M Eski, I Sahin, S Ilgan, S Isik, et al. (2010) Saving the zone of stasis in burns with activated protein C: an experimental study in rats. Burns 36(3): 397-402.

6. Kagan RJ, MD Peck, DH Ahrenholz, WL Hickerson, J Holmes IV, et al. (2013) Surgical management of the burn wound and use of skin substitutes: an expert panel white paper. J Burn Care Res 34(2): e60-e79.

7. Saffle JR, B Davis, P Williams, ABARP Group (1995) Recent outcomes in the treatment of burn injury in the United States: a report from the American Burn Association Patient Registry. The J burn care rehabilitation 16(suppl 1): 219-232.

8. Lionelli G, E Pickus, O Beckum, R Decoursey, R Korentager, et al. (2005) A three-decade analysis of factors affecting burn mortality in the elderly. Burns 31(8): 958-963.

9. Fitzwater J, GF Purdue, JL Hunt, GE OKeefe (2003) The risk factors and time course of sepsis and organ dysfunction after burn trauma. Journal of Trauma and Acute Care Surgery 54(5): 959-966.

10. Barrow RE, MSpies, LN Barrow, DN Herndon (2004) Influence of demographics and inhalation injury on burn mortality in children. Burns 30(1): $72-77$

11. Atiyeh BS, SW Gunn, SN Hayek (2005) State of the art in burn treatment World journal of surgery 29(2): 131-148.

12. Baker CC, CL Miller, DD Trunkey (1979) Predicting fatal sepsis in burn patients. The J trauma 19(9): 641-648.

13. Bang RL, PN Sharma, SC Sanyal, I Al Najjadah (2002) Septicaemia after burn injury: a comparative study. Burns 28(8): 746-751.

14. Tiwari V (2012) Burn wound: How it differs from other wounds? Indian journal of plastic surgery: official publication of the Association of Plastic Surgeons of India 45(2): 364.
15. Gurtner GC, S Werner, Y Barrandon, MT Longaker (2008) Wound repair and regeneration. Nature 453(7193): 314.

16. Werner S, T Krieg, H Smola (2007) Keratinocyte-fibroblast interactions in wound healing. J Investigative Dermatology 127(5): 998-1008.

17. Santajit S, N Indrawattana (2016) Mechanisms of antimicrobial resistance in ESKAPE pathogens. BioMed research international 2016.

18. Alebachew T, G Yismaw, A Derabe, Z Sisay (2012) Staphylococcus aureus burn wound infection among patients attending Yekatit 12 hospital burn unit, Addis Ababa, Ethiopia. Ethiopian j health sciences 22(3): 209-213.

19. Alharbi SA, M Zayed (2014) Antibacterial susceptibility of bacteria isolated from burns and wounds of cancer patients. J Saudi Chemical Society 18(1): 3-11.

20. Ronat JB, J Kakol, MN Khoury, M Berthelot, O Yun, et al. (2014) Highly drug-resistant pathogens implicated in burn-associated bacteremia in an Iraqi burn care unit. PloS one 9(8): e101017.

21. Melake NA, NA Eissa, TF Keshk, AS Sleem (2015) Prevalence of multidrug-resistant bacteria isolated from patients with burn infection. Menoufia Med J 28(3): 677-684.

22. Ramakrishnan M, SP Bai, M Babu (2016) Study on biofilm formation in burn wound infection in a pediatric hospital in Chennai, India. Annals of burns and fire disasters 29(4): 276-280.

23. Lacey R, J Grinsted (1973) Genetic analysis of methicillin-resistant strains of Staphylococcus aureus; evidence for their evolution from a single clone. Journal of Medical Microbiology 6(4): 511-526.

24. Kuroda M, T Ohta, I Uchiyama, T Baba, H Yuzawa, et al. (2001) Whole genome sequencing of meticillin-resistant Staphylococcus aureus. The Lancet 357(9264): 1225-1240.

25. Crowcroft NS, O Ronveaux, DL Monnet, R Mertens (1999) Methicillinresistant Staphylococcus aureus and antimicrobial use in Belgian hospitals. Infection Control Hospital Epidemiology 20(1): 31-36.

26. Hori S, R Sunley, A Tami, H Grundmann (2002) The Nottingham Staphylococcus aureus population study: prevalence of MRSA among the elderly in a university hospital. J Hospital Infection 50(1): 25-29.

27. Chambers HF (1997) Methicillin resistance in staphylococci: molecular and biochemical basis and clinical implications. Clinical microbiology reviews 10(4): 781-791.

28. Lindenmayer JM, S Schoenfeld, RO grady, JK Carney (1998) Methicillinresistant Staphylococcus aureus in a high school wrestling team and the surrounding community. Archives of Internal Medicine 158(8): 895-899.

29. Lina G, Y Piémont, F Godail Gamot, M Bes, MO Peter, et al. (1999) Involvement of Panton-Valentine leukocidin-producing Staphylococcus aureus in primary skin infections and pneumonia. Clinical Infectious Diseases 29(5): 1128-1132.

30. Kloos WE, MS Musselwhite (1975) Distribution and persistence of Staphylococcus and Micrococcus species and other aerobic bacteria on human skin. Applied microbiology 30(3): 381-395.

31. Wertheim HF, DC Melles, MC Vos, W van Leeuwen, A van Belkum, et al. (2005) The role of nasal carriage in Staphylococcus aureus infections. The Lancet infectious diseases 5(12): 751-762.

32. Corbella X, M Domíguez, M Pujol, J Ayats, M Sendra, et al. (1997) Staphylococcus aureus nasal carriage as a marker for subsequent staphylococcal infections in intensive care unit patients. European Journal of Clinical Microbiology and Infectious Diseases 16(5): 351-357.

33. Luzar MA, GA Coles, B Faller, A Slingeneyer, GD Dah, et al. (1990) Staphylococcus aureus nasal carriage and infection in patients on continuous ambulatory peritoneal dialysis. New England J Medicine 322(8): 505-509.

34. Williams JV, BR Vowels, PJ Honig, JJ Leyden (1998) S. aureus isolation from the lesions, the hands, and the anterior nares of patients with atopic dermatitis. Pediatric dermatology 15(3): 194-198. 
35. Diekema D, M Pfaller, F Schmitz, J Smayevsky, J Bell, et al. (2001) Survey of infections due to Staphylococcus species: frequency of occurrence and antimicrobial susceptibility of isolates collected in the United States, Canada, Latin America, Europe, and the Western Pacific region for the SENTRY Antimicrobial Surveillance Program, 1997-1999. Clinical Infectious Diseases 32(Suppl 2): S114-S132.

36. Lyytikäinen O, J Lumio, H Sarkkinen, E Kolho, A Kostiala, et al. (2002) Nosocomial bloodstream infections in Finnish hospitals during 19992000. Clinical Infectious Diseases 35(2): e14-e19.

37. Eggimann P, D Pittet (2002) Overview of catheter-related infections with special emphasis on prevention based on educational programs. Clinical microbiology and Infection 8(5): 295-309.

38. Dings MM, PM Orwin, P Schlievert (2000) Exotoxins of Staphyloccocus aureus. Clin Microbiol Rev 13: 16-34.

39. Drews TD, JL Temte, BC Fox (2006) Community-associated methicillinresistant Staphylococcus aureus: review of an emerging public health concern. WMJ MADISON 105(1): 52-57.

40. Deshpande LM, TR Fritsche, RN Jones (2004) Molecular epidemiology of selected multidrug-resistant bacteria: a global report from the SENTRY Antimicrobial Surveillance Program. Diagnostic microbiology and infectious disease 49(4): 231-236.

41. Bradley SF (1997) Methicillin-resistant Staphylococcus aureus in nursing homes. Drugs \& aging 10(3): 185-198.

42. Kuikka A, VV Valtonen (1994) Improved outcome of Staphylococcus aureus bacteremia. Infectious Diseases in Clinical Practice 3(4): 282287.

43. INDUE Y, S Kohno, T Fujii, T Otsubo, N Mori, et al. (1995) Clinica evaluation of catheter-related fungemia and bacteremia. Internal medicine 34(6): 485-490.

44. Fridkin SK, JC Hageman, M Morrison, LT Sanza, K Como Sabetti, et al. (2005) Methicillin-resistant Staphylococcus aureus disease in three communities. New England Journal of Medicine 352(14): 1436-1444.

45. Fridkin SK, J Hageman, LK McDougal, J Mohammed, WR Jarvis, et al. (2003) Epidemiological and microbiological characterization of infections caused by Staphylococcus aureus with reduced susceptibility to vancomycin, United States, 1997-2001. Clinical Infectious Diseases 36(4): 429-439.

46. Klevens RM, JR Edwards, FC Tenover, LC McDonald, T Horan, et al. (2006) Changes in the epidemiology of methicillin-resistant Staphylococcus aureus in intensive care units in US hospitals, 1992-2003. Clinical infectious diseases 42(3): 389-391.

47. Johnson AP, A Pearson, G Duckworth (2005) Surveillance and epidemiology of MRSA bacteraemia in the UK. Journal of Antimicrobial Chemotherapy 56(3): 455-462

48. Björholt I, E Haglind (2004) Cost-savings achieved by eradication of epidemic methicillin-resistant Staphylococcus aureus (EMRSA)-16 from a large teaching hospital. European J Clinical Microbiology and Infectious Diseases 23(9): 688-695.

49. Hafiz S, A Hafiz, L Ali, A Chughtai, B Memon, et al. (2002) Methicillin resistant Staphylococcus aureus: a multicentre study. J Pak Med Assoc 52(7): 312-314.

50. Butt T, RN Ahmad, M Usman, A Mahmood (2004) Methicillin-resistant Staphylococcus aureus, Pakistan, 1996-2003. Emerging infectious diseases 10(9): 1691.

51. Hakim S, S Arshed, M Iqbal, S Javaid (2007) Vancomycin sensitivity of Staphylococcus aureus isolates from hospital patients in Karachi, Pakistan. Libyan Journal of Medicine 2(4): 176-179.

52. Ahmed K, A Mahmood, MK Ahmad, K Hussain (2007) Methicillin resistant Staphylococcus aureus prevalence amongst Community versus Hospital acquired skin and soft tissue infections. Infect Dis J Pak 16: 1416.
53. Burnie J, R Matthews, A Jiman Fatami, P Gottardello, S Hodgetts et al. (2000) Analysis of 42 cases of septicemia caused by an epidemic strain of methicillin-resistant Staphylococcus aureus: evidence of resistance to vancomycin. Clinical infectious diseases 31(3): 684-689.

54. Blot SI, KH Vandewoude, EA Hoste, FA Colardyn (2002) Outcome and attributable mortality in critically ill patients with bacteremia involving methicillin-susceptible and methicillin-resistant Staphylococcus aureus. Archives of Internal Medicine 162(19): 2229-2235.

55. Harbarth S, J Garbino, J Pugin, JA Romand, D Lew, et al. (2003) Inappropriate initial antimicrobial therapy and its effect on survival in a clinical trial of immunomodulating therapy for severe sepsis. The American journal of medicine 115(7): 529-535.

56. Kuehnert MJ, HA Hill, BA Kupronis, JI Tokars, SL Solomon, et al. (2005) Methicillin-resistant-Staphylococcus aureus hospitalizations, United States. Emerging infectious diseases 11(6): 468.

57. Zetola N, JS Francis, EL Nuermberger, WR Bishai (2005) Communityacquired meticillin-resistant Staphylococcus aureus: an emerging threat. The Lancet infectious diseases 5(5): 275-286.

58. Bartlett J (2004) Community-Acquired Methicillin-Resistant Staphylococcus aureus and Other Drug-Resistant Pathogens CME. Infectious Diseases Society of América. Anual Meeting.

59. Johnson LB, LD Saravolatz (2005) Community-acquired MRSA: current epidemiology and management issues. Infections in medicine 22(1): 1620.

60. Eftekhar F, S Moazami Goudarzi (2013) Assessment of carbapenem susceptibility and multidrug resistance in Pseudomonas aeruginosa burn isolates in Tehran. Jundishapur Journal of Microbiology 6(2): 162165 .

61. Ekrami A, E Kalantar (2007) Bacterial infections in burn patients at a burn hospital in Iran. Indian Journal of Medical Research 126(6): $541-$ 544 .

62. Arslan E, C Dalay, M Yavuz, L Göcenler, S Acartürk, et al. (1999) Gramnegative bacterial surveillance in burn patients. Proteus 95: 53.

63. Naqvi ZA, K Hashmi, QM Rizwan, SA Kharal (2005) Multidrug resistant Pseudomonas aeruginosa: a nosocomial infection threat in burn patients. Pak J Pharma 22(2): 9-15.

64. Ikpeme E, J Nfongeh, K Enyi Idoh, L Etim, F Akubuenyi, et al. (2013) Prevalence, antibiogram profile and cross transmission of Pseudomonas aeruginosa in a tertiary burn unit. Malaysian J Microbiology 9(1): 116119.

65. Asghar AH (2012) Antimicrobial susceptibility and metallo- $\beta$-lactamase production among Pseudomonas aeruginosa isolated from Makkah Hospitals. Pakistan Journal of Medical Sciences 28(5).

66. Upadhaya S, R Shenoy V Shetty, A Lamsal, P Lamichhane, S Pokhrel, et al. (2014) Multi-drug Resistant Pseudomonas aeruginosa Isolated from Intensive Care Burn Unit. Int J Biomed Res 5: 271-273.

67. Biswal I, BS Arora, N Dimple Kasana (2014) Incidence of multidrug resistant pseudomonas aeruginosa isolated from burn patients and environment of teaching institution. Journal of clinical and diagnostic research: JCDR 8(5): DC26.

68. Saaiq M, S Ahmad, MS Zaib (2015) Burn wound infections and antibiotic susceptibility patterns at Pakistan Institute of Medical Sciences, Islamabad, Pakistan. World journal of plastic surgery 4(1): 9-15.

69. Hassuna NA, AHI Mohamed, SM Abo Eleuoon, HAWA Rizk (2015) High prevalence of multidrug resistant Pseudomonas aeruginosa recovered from infected burn wounds in children. Archives of Clinical Microbiology $6(4)$.

70. Song Y, H Dou, X Li, X Zhao, Y Li, et al. (2017) Exosomal miR-146a Contributes to the Enhanced Therapeutic Efficacy of Interleukin-1 $\beta$ Primed Mesenchymal Stem Cells Against Sepsis. Stem Cells 35(5): 12081221. 


\section{ISSN: 2574-1241}

DOI: $10.26717 /$ BJSTR.2020.25.004222

Abolanle AA Kayode. Biomed J Sci \& Tech Res

(c) (P) This work is licensed under Creative BY Commons Attribution 4.0 License

Submission Link: https://biomedres.us/submit-manuscript.php

$\begin{array}{ll}\text { BIOMEDICAL } & \text { Assets of Publishing with us } \\ \text { RESEARCHES } & \text { - Global archiving of articles } \\ \text { - Immediate, unrestricted online access } \\ \text { - Rigorous Peer Review Process } \\ \text { https://biomedres.us/ }\end{array}$

